OSIPP OSIPP Discussion Paper : DP-2006-E-004-Rev

\title{
Contracting with Self-Esteem Concerns
}

November 15, 2006

\author{
J unichiro Ishida
}

Associate Professor, Osaka School of International Public Policy (OSI PP)

【キーワード】 Self-esteem, Bayesian learning, Tradeoff between risk and incentives, Contract

【要約】 It is widely accepted in social psychology that the need to maintain and enhance self-esteem is a fundamental human motive. We incorporate this factor into an otherwise ordinary principal-agent framework and examine its impact on the optimal incentive scheme and the agent's behavior, especially focusing on a form of intrapersonal strategy known as self-handicapping. Incorporating self-esteem concerns into a contracting situation yields an implication that goes against the conventional wisdom: the standard tradeoff between risk and incentives may break down in the presence of self-esteem concerns because uncertainty mitigates the need for self-handicapping, providing a potential reason for why we do not empirically observe this tradeoff in a robust manner. We characterize an intuitive condition for this anomaly to arise and present a set of testable implications. Along the way, we also show that the fragility of self-esteem (the variance) is just as important as its level (the mean) in selecting agents. Finally, this simple logic is applied to a team problem to show why and how people are better motivated under team production than under individual production.

I am grateful to Hideshi Itoh and seminar participants at CTW Summer Conference for hel pful discussion. Financial support from Grants-in-Aid for Young Scientists (B) is gratefully acknowledged. 


\section{Introduction}

In most economic analyses, agents are typically assumed to possess precise knowledge of their own attributes. As this assumption symbolically indicates, economists have paid relatively less attention to potential consequences of learning about oneself. This aspect of economics perhaps draws clear contrast with psychology where how people come to understand themselves in general, or the issue of self-esteem in particular, has always been the topic of utmost concern. Although self-esteem is defined in a variety of ways, including perceived ability and competence, physical attractiveness and interpersonal relations, it is now widely accepted in social psychology that the need to maintain and enhance self-esteem is a fundamental human motive (Leary and Downs, 1995).

Recently, there is also a growing amount of interest on the issues of self-esteem concerns among economists, as exemplified most notably by Benabou and Tirole (2002). In this paper, we explore implications of self-esteem concerns in a contracting situation by following this line of research from a different perspective. To this end, we start our analysis with two key presumptions: (i) the agent in question is a rational Bayesian learner, but with vague knowledge about himself to begin with; and (ii) he benefits directly from having a positive image of himself, for various reasons. Under this setup, an outcome of some action, which becomes a signal of his own unknown attributes, has a direct effect on the payoffs, aside from compensations from the agreed-upon contract. The presence of selfesteem concerns has a critical bite especially when ability and effort are complementary to each other, as is often assumed in many economic analyses. Under this condition, the agent actually stretches the distribution of outcome by exerting effort, thereby facilitating the learning process about oneself. Conversely speaking, by not exerting effort and hence compressing the distribution of outcome, the failure becomes relatively insignificant as the agent can still remain vague about his own attributes: even if the task turns out to be unsuccessful, the agent can attribute it to the lack of effort rather than the lack of ability.

An especially interesting situation arises when the agent is averse to having a negative self-image or, more precisely, the utility gain from self-esteem is concave in its level. In such a case, given 
the same mean, the agent is actually made better off by not obtaining more information and hence remaining vague about himself. This motive gives rise to a form of intrapersonal strategy, known as self-handicapping in social psychology, where individuals create impediments to successful performances. In some instances, self-handicapping takes a highly active form where they take performanceimpairing drugs or alcohol before the achievement activity such as the exam $;^{1}$ in others, it takes a more passive form where they intentionally withhold effort in order to make failure meaningless (e.g., not studying hard before the exam). In either case, people resort to self-handicapping as a strategy to shield themselves from 'facing the truth' that can sometimes be painful. In this paper, we mostly focus on the role of the latter form of (passive) self-handicapping in a principal-agent framework. ${ }^{2}$

Incorporating self-esteem concerns into a contracting situation yields an implication that goes against the conventional wisdom. The central tenet of the contract theory is the tradeoff between risk and incentives, which asserts that the cost of inducing any given level of effort generally increases with the extent of uncertainty faced by the agent. This general conclusion suggests that, because the agency cost increases with the uncertainty of the underlying environment, incentive pays would be muted in risky situations. Despite its clear prediction, however, finding this tradeoff in actual data has turned out to be a daunting task, as emphasized in a series of papers by Prendergast (2000, 2002a, $2002 b) .^{3}$

In those papers, Prendergast provides several plausible reasons why we may not observe this tradeoff by focusing on the neglected impact of uncertainty on various aspects of monitoring behavior (Prendergast, 2000, 2002b) or the allocation of responsibility (Predergast, 2002a). ${ }^{4}$ The basic stance there is that there are some hidden aspects of uncertainty that tend to be overlooked, and those aspects influence the optimal form of incentive structure. In contrast, this paper points out a channel through

\footnotetext{
${ }^{1}$ For instance, Tucker, Vuchinich and Sobell (1981) observe that college students who are not sure to perform well at an intellectual task volunteer to drink alcohol before the task.

${ }^{2}$ In what follows, we refer to self-handicapping simply as an act of withholding effort in an attempt to obscure one's own attributes.

${ }^{3}$ See Jin (2002) and Core and Guay (2002) for more recent empirical evidence.

${ }^{4}$ Several alternative explanations have also been suggested. For instance, Ackerberg and Botticini (2002) argue that the standard tradeoff may disappear if endogenous matching is explicitly considered. Raith (2003) emphasizes the impact of market competition on the provision of managerial incentives.
} 
which the presence of uncertainty itself directly yields a positive incentive effect which weakens or even breaks down the standard tradeoff..$^{5}$ The main contention of this paper is that uncertainty may yield a positive incentive effect because the uncertainty associated with the task obscures the agent's true worth, thereby reducing the need for self-handicapping. Since the need for self-handicapping naturally increases the agency cost to induce any given level of effort, an increase in uncertainty may actually decrease the agency cost; this is so even when the agent in question is a rational Bayesian learner with no ability to manipulate information. Along the way, our analysis, and especially the reasoning mentioned above, give rise to a set of testable implications on the tradeoff between risk and incentives.

As an application of this logic, we also make a point that this mechanism explains why team production can be more profitable, compared to individual production. As a recent trend, many firms start adopting team production as a way to motivate workers. This trend is somewhat puzzling because the standard theory asserts that team production invites a notorious free-rider problem because each agent's contribution becomes less distinguishable in teams. To account for this trend, several explanations have been offered, from the presence of complementarities to the importance of repeated interactions (e.g., Che and Yoo, 2001; Ishida, 2006). This paper provides yet another explanation for why team production can be profitable based on self-esteem concerns. In short, team production can be profitable because the outcome of some team projects is less reflexive of one's own attributes whereas the outcome more accurately reflects them under individual production. Since the need for self-handicapping is lower under team production, the cost of inducing effort can also be lower, consequently making team production more profitable. Our framework thus provides an explanation for why workers are better motivated under team production, especially to the extent that can overcome the free-rider problem - the psychological side of team production often emphasized by managers and executives.

As already mentioned, there is a growing body of literature which incorporates psychological factors

\footnotetext{
${ }^{5}$ Our stance here is that the presence of self-esteem concerns can provide one factor, possibly among many others, which can break down the standard tradeoff. It should thus be noted that the purpose of this paper is simply to complement, but certainly not to deny, the existing views on this issue.
} 
into formal economic analyses. There are largely two strands of literature that explore consequences of learning about oneself. The first strand deals with interpersonal situations, which can be seen as a particular type of the informed-principal problem. For instance, Benabou and Tirole (2003) analyze how disclosing information about the agent (or his surrounding environment) might affect his 'intrinsic motivation. ${ }^{6}$ Ishida (2006) further extends this idea and analyzes optimal promotion rules when the agent is ambiguous about his own attributes and gains some information about himself from the principal's behavior.

The second strand, on the other hand, deals with intrapersonal situations. Economic consequences of self-esteem concerns are broadly analyzed by Benabou and Tirole (2002), focusing mainly on problems that stem from time-inconsistent preferences along the line of Carrillo and Mariotti (2000). Using intrapersonal games, they analyze issues such as why self-confidence can be beneficial and how the acquisition of information alters the behavior of future selves. Benabou and Tirole (2004) combine imperfect recall with time-inconsistent preferences and develop a theory of personal rules arising from self-reputation concerns. Within a framework that is similar to ours (unbiased Bayesian learning and self-esteem concerns), Koszegi (2006) shows how self-esteem concerns give rise to biased beliefs even for rational Bayesian learners and distort the choice of task. The present paper belongs to this second strand where the agent gains some information about himself through his own actions (self-signaling). As a departure from previous studies, we focus on a contracting situation and analyze how self-esteem concerns affect the optimal incentive scheme, especially in its relation to the tradeoff between risk and incentives. $^{7}$

The present setup is also closely related to models of career concerns, most notably by Holmstrom (1999), in that it deals with dynamic signaling incentives. ${ }^{8}$ The difference from this strand of literature is subtle yet critical. In models of career concerns, signaling is directed to outside observers who only

\footnotetext{
${ }^{6}$ Benabou and Tirole (2006) consider a different context where an agent possesses private information about his tastes and attempts to signal them in order to appear 'prosocial,' and show how this reputation effect is contaminated by extrinsic rewards.

${ }^{7}$ There is recently an increasing number of works that incorporate behavioral factors into contracting situations. Some examples along this line include Itoh (2004), Grund and Sliwka (2005) and Englmaier and Wambach (2006) just to name a few.

${ }^{8}$ Also, see Dewatripont, Jewitt and Tirole (1999a,1999b)
} 
have a limited set of information about the sender. Career concerns then typically yield a positive incentive effect because the agent can gain nothing by withholding effort when outside observers expect him to do otherwise. This draws clear contrast to the present framework where the focus is placed on self-signaling and the agent's information set, on which the posterior belief is conditioned, naturally includes his own actions. As we will see shortly, this seemingly slight difference amounts to different implications where self-esteem concerns actually yield a negative incentive effect under some conditions, ultimately working to counteract with the standard tradeoff between risk and incentives.

The paper proceeds as follows. Section 2 briefly discusses why we care about self-esteem as our entire analysis rests on this presumption. Section 3 illustrates the environment where we introduce self-esteem concerns into an otherwise ordinary principal-agent model. Section 4 derives the main results and shows that self-esteem concerns have an effect that tends to negate the standard tradeoff between risk and incentives. Section 5 extends the model and shows that the same logic also works for team production, providing an explanation for why team production can be so profitable. Finally, section 6 makes some concluding remarks.

\section{Why do we care about self-esteem?}

Before we proceed to the formal analysis, we first briefly discuss why we care about our self-images at all because our analysis hinges crucially on the presumption that people benefit from having a positive image of themselves. It is argued, mostly in psychology, that one of the most basic human responses when faced with some object is evaluation (Tesser and Martin, 1996). It is then only natural that since the most important object in one's life is arguably the self, the evaluation of self, or self-esteem, has been the central theme in social psychology from the very beginning (Tesser, 2001). Consequently, this voluminous literature has produced a set of theories and hypotheses on why there is ever a need for a positive view of self.

On the demand side for self-esteem, Benabou and Tirole (2002) cite three possible factors on why we prefer to have a positive view of ourselves. The first factor is what they refer to as the 
consumption value of self-esteem: we simply like to consider ourselves as able, attractive, caring and so on. A possible channel through which the consumption value arises is a connection between selfesteem and affect that is well recognized in social psychology (Tesser and Collins, 1988): a rise in self-esteem is often associated with positive affect while a decrease is associated with negative affect. This then implies that the need for self-esteem inherently arises when we have preferences for positive affect. According to this view, self-esteem is just another argument in the utility function, along with the consumption of private goods. ${ }^{9}$

The second factor is the motivation value of self-esteem which becomes valuable especially in situation where our willpower is tested periodically. Evidence in favor of this view is fairly abundant as it is often observed that high self-esteem helps people achieve their goals. For instance, there is a tendency that people with high self-esteem perform better and persist longer at tasks (Bandura, 1977). In fact, people with high self-esteem tend to persist longer even at impossible tasks (McFarlin, Baumeister and Blascovich, 1984). Here, the need for self-esteem arises indirectly where high selfesteem is instrumental in inducing better performances which are expected to lead to higher payoffs in future transactions.

Finally, high self-esteem can be valuable in interpersonal situations, which Benabou and Tirole (2002) label as the signaling value of self-esteem. The idea is that it is easier to convince others that a person has some favorable traits when he or she in fact possesses such qualities. In a world full of information asymmetry, the ability to present ourselves favorably to others can be a valuable asset in situations such as job interviews, business negotiations, and so on. Somewhat related to this is Barkow's (1980) notion that self-esteem is an indicator of dominance in a group. According to this view, high self-esteem implies dominance in a group, which certainly helps convincing others.

While it is undoubtedly crucial to examine the origins of self-esteem concerns, we do not take any specific position about which view is more appropriate in this paper, in order to focus our attention

\footnotetext{
${ }^{9}$ The consumption value of self-esteem can also be explained by the terror management theory (TMT). According to Greenberg, Pyszczynski and Solomon (1986), the awareness of death, and its finality, produces terror. Being a part of one's culture reduces this terror since the culture lives on forever and also promises an afterlife. Self-esteem becomes important in this sense as it is an indicator of the extent to which the individual is meeting cultural standards.
} 
to the impact of self-esteem concerns on the optimal incentive scheme. In what follows, therefore, we simply assume that the agent in question benefits from having a positive image of himself for some unspecified reasons. More precisely, the present framework should be interpreted as adopting the first approach (the consumption value) or some reduced form of the other two (the motivation value and the signaling value) for practical purposes.

\section{The model}

We illustrate our contention by a simple model of moral hazard, with discrete effort choices and linear contracts. ${ }^{10}$ The main purpose here is to make a point that the agency cost to induce any given level of effort may decrease with an increase in the extent of uncertainty of the underlying environment, quite contrary to the conventional wisdom.

Consider a model with a principal (female) and an agent (male). The agent is characterized by the ability type $\eta \sim N\left(\mu, \sigma_{\eta}^{2}\right), \mu>0$, which is initially not known to anyone, including the agent himself. The prior distribution of the ability type is common knowledge. Under this setup, the mean $\mu$ (the prior belief on the agent's ability type) reflects the agent's initial self-esteem level while the variance $\sigma_{\eta}^{2}$ reflects its fragility. We hence regard that the agent is more secure (fragile) about his own self-images when $\sigma_{\eta}^{2}$ is relatively small (large). When $\sigma_{\eta}^{2}=0$, the agent is certain about his own attribute, and the problem converges to the standard case.

The principal lets the agent engage in a task where the agent's effort level is denoted by $e \in\{0,1\}$. The cost of exerting effort is given by $c(e)$ where $c(0)=0$ and $c(1)=c$. The observable outcome $y \in R$ is a noisy signal of ability and effort, specified as

$$
y=\eta e+\varepsilon,
$$

where $\varepsilon \sim N\left(0, \sigma_{\varepsilon}^{2}\right)$ is a disturbance distributed independently of $\eta$. Among other things, this specification implies that ability and effort are complementary to each other. The variance of the disturbance

\footnotetext{
${ }^{10}$ Although it is in principle possible and conceptually straightforward to extend the model to continuous effort choices, the derivation of optimal contracts can be quite complicated computationally. The current specification is simple and tractable enough (although still computationally highly tedious) to succinctly make our point.
} 
$\sigma_{\varepsilon}^{2}$ measures the extent of uncertainty faced by the agent in this economy. Our ultimate concern is its impact on the cost of inducing effort $e=1$.

We assume that the outcome is the only contractible variable in this environment and focus on a linear contract contingent on it. More precisely, the principal offers

$$
w=\beta y+\gamma,
$$

where $\gamma$ denotes some (constant) transfer payment. Given this, the contract can generically be written as $(\beta, \gamma)$.

Finally, we need to specify the agent's preferences, which mark the sole departure from the conventional setup. While the agent gains utility from material benefits as usual, he also has vested interests in his own ability type. Let $\tilde{\mu}$ denote the agent's posterior belief about his own ability type, which is contingent on the effort level $e$ and the outcome $y$. The agent's preferences are specified by the following CARA utility function:

$$
u(w-c(e)+\alpha(\tilde{\mu}-\mu))=-\exp \{-r[w-c(e)+\alpha(\tilde{\mu}-\mu)]\}, r>0 .
$$

Throughout the analysis, we broadly refer to the last term $\alpha(\tilde{\mu}-\mu)$ as the self-esteem concern, and the parameter $\alpha \geq 0$ measures its relative strength. When $\alpha=0$, the self-esteem concern is totally absent, and the agent's utility depends solely on material payoffs as is normally assumed.

The timing of the model is summarized as follows:

stage 1: The principal offers a contract $(\beta, \gamma)$. If the agent rejects it, both receive the reservation payoff, which is normalized to zero.

stage 2: The agent determines the effort level $e$.

stage 3: The outcome $y$ is realized and the wage is paid as specified by the agreed-upon contract. 


\section{The optimal contract with self-esteem concerns}

\subsection{A tenuous tradeoff between risk and incentives}

The crux of the model is how the agent's behavior influences the extent of uncertainty that he faces. Let $\pi:=w-c(e)+\alpha(\tilde{\mu}-\mu)$ denote the total net payoff. Then, the certainty-equivalent (CE) version of the agent's problem is defined as

$$
\max _{e \in\{0,1\}} \quad \pi_{\mathrm{CE}}:=\beta \mu e+\gamma-c(e)-\frac{r}{2} \operatorname{var}(\pi \mid e) .
$$

Note that $E(\tilde{\mu} \mid e)=\mu$ regardless of the agent's effort choice $e$. The agent exerts effort if and only if

$$
\beta \mu \geq c+\frac{r}{2}[\operatorname{var}(\pi \mid e=1)-\operatorname{var}(\pi \mid e=0)]
$$

Provided that the agent's reservation payoff is normalized to zero, the participation constraint is given by

$$
\beta \mu e+\gamma \geq c(e)+\frac{r}{2} \operatorname{var}(\pi \mid e)
$$

when the contract offered is designed to induce effort $e$. Define $w_{e}\left(\sigma_{\eta}^{2}, \sigma_{\varepsilon}^{2}\right):=c(e)+(r / 2) \operatorname{var}(\pi \mid e)$ as the agency cost of inducing effort $e$. We are generally interested in how $w_{1}$ is related to an increase in the extent of uncertainty $\sigma_{\varepsilon}^{2}$.

To explicitly solve this problem, we obviously need to obtain the conditional variance of the payoff $\operatorname{var}(\pi \mid e)$. For this, we can obtain the following result which holds even if $e$ can be chosen continuously over $[0, \infty)$.

\section{Lemma 1}

$$
\operatorname{var}(\pi \mid e)=\left(\beta+\frac{\alpha e \sigma_{\eta}^{2}}{\sigma_{\varepsilon}^{2}+e^{2} \sigma_{\eta}^{2}}\right)^{2}\left(e^{2} \sigma_{\eta}^{2}+\sigma_{\varepsilon}^{2}\right):=\Gamma_{e}^{2}\left(e^{2} \sigma_{\eta}^{2}+\sigma_{\varepsilon}^{2}\right),
$$

for any given $e \in[0, \infty)$.

Proof: We proceed here as if the effort choice is continuous for more generality. The outcome $y$ in this environment contains some information about the agent's ability type. The best estimate from 
that observation alone is given by $\hat{\eta}:=y / e=(\eta e+\varepsilon) / e .^{11}$ Note that, given the true $\eta, \hat{\eta}$ is distributed normally with precision $e^{2} / \sigma_{\varepsilon}^{2}$. The agent then updates his prior with this new information, and the posterior belief is hence given by

$$
\tilde{\mu}=\frac{\frac{1}{\sigma_{\eta}^{2}} \mu+\frac{e^{2}}{\sigma_{\varepsilon}^{2}} \hat{\eta}}{\frac{1}{\sigma_{\eta}^{2}}+\frac{e^{2}}{\sigma_{\varepsilon}^{2}}}=\frac{\sigma_{\varepsilon}^{2} \mu+e^{2} \sigma_{\eta}^{2} \hat{\eta}}{\sigma_{\varepsilon}^{2}+e^{2} \sigma_{\eta}^{2}}=\frac{\sigma_{\varepsilon}^{2} \mu+e^{2} \sigma_{\eta}^{2} \eta+e \sigma_{\eta}^{2} \varepsilon}{\sigma_{\varepsilon}^{2}+e^{2} \sigma_{\eta}^{2}}
$$

See DeGroot $($ ch.9, 1970) for more detail. It then follows from this that the agent's ex post payoff is

$$
\pi=\beta(\eta e+\varepsilon)+\gamma-c(e)+\alpha \frac{e^{2} \sigma_{\eta}^{2}(\eta-\mu)+e \sigma_{\eta}^{2} \varepsilon}{\sigma_{\varepsilon}^{2}+e^{2} \sigma_{\eta}^{2}} .
$$

Since $\alpha, \beta, \gamma$ and $\mu$ are all constant at this stage, we have

$$
\operatorname{var}(\pi \mid e)=\left(\beta e+\frac{\alpha e^{2} \sigma_{\eta}^{2}}{\sigma_{\varepsilon}^{2}+e^{2} \sigma_{\eta}^{2}}\right)^{2} \sigma_{\eta}^{2}+\left(\beta+\frac{\alpha e \sigma_{\eta}^{2}}{\sigma_{\varepsilon}^{2}+e^{2} \sigma_{\eta}^{2}}\right)^{2} \sigma_{\varepsilon}^{2}=\left(\beta+\frac{\alpha e \sigma_{\eta}^{2}}{\sigma_{\varepsilon}^{2}+e^{2} \sigma_{\eta}^{2}}\right)^{2}\left(e^{2} \sigma_{\eta}^{2}+\sigma_{\varepsilon}^{2}\right) .
$$

Q.E.D.

Several remarks are in order. First, as $\sigma_{\eta}^{2} \rightarrow 0$, the problem evidently converges to the standard case where $\operatorname{var}(\pi \mid e)=\beta^{2} \sigma_{\varepsilon}^{2}$ regardless of $e$, and the incentive compatibility constraint is naturally reduced to $\beta \mu \geq c$. A decrease in $\alpha$, on the other hand, yields a slightly different implication: as $\alpha \rightarrow 0$, i.e., as the self-esteem concern ceases to exist, the self-esteem fragility $\sigma_{\eta}^{2}$ is just another form of uncertainty, playing quite a similar role to $\sigma_{\varepsilon}^{2} \cdot{ }^{12}$ When both $\alpha>0$ and $\sigma_{\eta}^{2}>0$, however, there arises some interaction between them that works to negate the standard tradeoff between risk and incentives under some conditions.

It follows from lemma 1 that the incentive compatibility constraint can be written as

$$
\beta \mu \geq c+\frac{r}{2}\left[\Gamma_{1}^{2}\left(\sigma_{\eta}^{2}+\sigma_{\varepsilon}^{2}\right)-\Gamma_{0}^{2} \sigma_{\varepsilon}^{2}\right]
$$

Define $\beta^{*}$ as the minimum incentive needed to induce $e=1$, which satisfies (9) with equality. The next result establishes that there exists some nonempty interval of $\beta$ that can satisfy (9) when the prior belief $\mu$ is sufficiently large.

\footnotetext{
${ }^{11}$ Note that $\hat{\eta}$ is not well-defined when $e=0$. We know, however, that the posterior belief always coincides with the prior and its variance is zero when $e=0$. We thus proceed as if it is well-defined at the moment and later verify that the derived solution is indeed what we need to obtain.

${ }^{12}$ The only difference is that this uncertainty is complementary to the effort level so that more effort amplifies the extent of uncertainty. It is important to emphasize, however, that its impact is independent of $\sigma_{\varepsilon}^{2}$.
} 
Lemma 2 Suppose that $\mu$ is large enough to satisfy

$$
\left(\mu-\alpha r \sigma_{\eta}^{2}\right)^{2}>r \sigma_{\eta}^{2}(r \Phi+2 c)
$$

where

$$
\Phi:=\frac{\left(\alpha \sigma_{\eta}\right)^{2}}{\sigma_{\eta}^{2}+\sigma_{\varepsilon}^{2}} .
$$

There then exists some nonempty interval of $\beta$ that can satisfy (9), and the optimal contract $\left(\beta^{*}, \gamma^{*}\right)$ is given by

$$
\beta^{*}\left(\sigma_{\eta}^{2}, \sigma_{\varepsilon}^{2}\right)=\frac{\mu-\alpha r \sigma_{\eta}^{2}-\sqrt{\left(\mu-\alpha r \sigma_{\eta}^{2}\right)^{2}-r \sigma_{\eta}^{2}(r \Phi+2 c)}}{r \sigma_{\eta}^{2}}, \quad \gamma^{*}\left(\sigma_{\eta}^{2}, \sigma_{\varepsilon}^{2}\right)=\frac{r}{2} \beta^{* 2} \sigma_{\varepsilon}^{2} .
$$

Proof: It follows from (9) and lemma 1 that the minimum incentive needed to induce $e=1$, denoted as $\beta^{*}$, must solve

$$
\begin{aligned}
\beta^{*} & \left.=\frac{c}{\mu}+\frac{r}{2 \mu}\left[\left(\beta^{*}+\frac{\alpha \sigma_{\eta}^{2}}{\sigma_{\varepsilon}^{2}+\sigma_{\eta}^{2}}\right)^{2}\left(\sigma_{\eta}^{2}+\sigma_{\varepsilon}^{2}\right)-\beta^{* 2} \sigma_{\varepsilon}^{2}\right)\right] \\
& =\frac{c}{\mu}+\frac{r}{2 \mu}\left[\sigma_{\eta}^{2} \beta^{* 2}+\frac{2 \alpha \sigma_{\eta}^{2}\left(\sigma_{\eta}^{2}+\sigma_{\varepsilon}^{2}\right)}{\sigma_{\varepsilon}^{2}+\sigma_{\eta}^{2}} \beta^{*}+\frac{\left(\alpha \sigma_{\eta}^{2}\right)^{2}}{\sigma_{\varepsilon}^{2}+\sigma_{\eta}^{2}}\right] .
\end{aligned}
$$

Note that the righthand side is always positive, meaning that the roots, if they exist, must be positive. When $\mu$ is sufficiently large, there exist two positive roots, of which the smaller one constitutes the solution for our purpose. Applying the quadratic formula we obtain the optimal incentive as follows:

$$
\beta^{*}\left(\sigma_{\eta}^{2}, \sigma_{\varepsilon}^{2}\right)=\frac{\mu-\alpha r \sigma_{\eta}^{2}-\sqrt{\left(\mu-\alpha r \sigma_{\eta}^{2}\right)^{2}-r \sigma_{\eta}^{2}(r \Phi+2 c)}}{r \sigma_{\eta}^{2}},
$$

where $\Phi:=\left(\alpha \sigma_{\eta}\right)^{2} /\left(\sigma_{\eta}^{2}+\sigma_{\varepsilon}^{2}\right)$. It is evident from this that there exist two positive roots, and hence the solution is well-defined, when

$$
\left(\mu-\alpha r \sigma_{\eta}^{2}\right)^{2}>r \sigma_{\eta}^{2}(r \Phi+2 c) .
$$

Finally, since the participation constraint must also hold with equality, we obtain

$$
\gamma^{*}\left(\sigma_{\eta}^{2}, \sigma_{\varepsilon}^{2}\right)=\frac{r}{2} \operatorname{var}(\pi \mid e=0)=\frac{r}{2} \beta^{* 2} \sigma_{\varepsilon}^{2} .
$$

Q.E.D. 
In what follows, we restrict attention to the case where $\mu$ is large enough to satisfy the condition in the lemma, so that the optimal solution $\beta^{*}$ exists. With this well-defined solution, the agency cost of inducing $e=1$ can be written as

$$
w_{1}\left(\sigma_{\eta}^{2}, \sigma_{\varepsilon}^{2}\right)=c+\frac{r}{2} \Gamma_{1}^{2}\left(\sigma_{\eta}^{2}+\sigma_{\varepsilon}^{2}\right)=c+\frac{r}{2}\left[\beta^{*}\left(\sigma_{\eta}^{2}, \sigma_{\varepsilon}^{2}\right)+\frac{\alpha \sigma_{\eta}^{2}}{\sigma_{\varepsilon}^{2}+\sigma_{\eta}^{2}}\right]^{2}\left(\sigma_{\eta}^{2}+\sigma_{\varepsilon}^{2}\right) .
$$

Given this, we first make the following observation that the agency cost generally increases with $\sigma_{\varepsilon}^{2}$ under the conventional setup.

Proposition 1 The agency cost $w_{1}$ increases with the extent of uncertainty $\sigma_{\varepsilon}^{2}$ if $\alpha=0$ and/or $\sigma_{\eta}^{2}=0$

ProOF: If $\sigma_{\eta}^{2}=0$, the incentive compatibility constraint is simply reduced to $\beta \mu \geq c$ so that $\beta^{*}=c / \mu$. Note also that $\Gamma_{1}=\beta^{*}=c / \mu$ under this condition. The proposition is then immediately obtained from this.

If $\alpha=0$, the incentive compatibility constraint becomes $\beta \mu \geq c+\beta^{2} \sigma_{\eta}^{2}$. Again, the optimal $\beta^{*}$ is independent of $\sigma_{\varepsilon}^{2}$. Since $\Gamma_{1}=\beta^{*}$ as above, the agency cost increases with $\sigma_{\varepsilon}^{2}$, provided that a well-defined solution $\beta^{*}$ exists.

Q.E.D.

This conclusion that generally and robustly holds true in the conventional setup may be overturned because an increase in uncertainty obscures the self-evaluation process, mitigating the need for selfhandicapping. More precisely, we make the following claim which identifies the necessary and sufficient condition for this reversal to occur.

Lemma 3 Let $\partial \operatorname{var}(\pi \mid e=1) / \partial \sigma_{\varepsilon}^{2}$ denote the partial derivative with respect to $\sigma_{\varepsilon}^{2}$ when $\beta^{*}$ is held constant where

$$
\frac{\partial \operatorname{var}(\pi \mid e=1)}{\partial \sigma_{\varepsilon}^{2}}=\Gamma_{1}\left[\beta^{*}-\frac{\alpha \sigma_{\eta}^{2} \sigma_{\varepsilon}^{2}}{\left(\sigma_{\varepsilon}^{2}+\sigma_{\eta}^{2}\right)^{2}}\right]
$$

The agency cost $w_{1}$ then decreases with the extent of uncertainty $\sigma_{\varepsilon}^{2}$ if and only if

$$
\frac{\partial \operatorname{var}(\pi \mid e=1)}{\partial \sigma_{\varepsilon}^{2}}+2 \Gamma_{1}\left(\sigma_{\eta}^{2}+\sigma_{\varepsilon}^{2}\right) \frac{\partial \beta^{*}}{\partial \sigma_{\varepsilon}^{2}}<0 .
$$


PROOF: It is straightforward to obtain that

$$
\frac{\partial w_{1}}{\partial \sigma_{\varepsilon}^{2}}=\frac{r}{2} \frac{\partial \operatorname{var}(\pi \mid e=1)}{\partial \sigma_{\varepsilon}^{2}}+r \Gamma_{1}\left(\sigma_{\eta}^{2}+\sigma_{\varepsilon}^{2}\right) \frac{\partial \beta^{*}}{\partial \sigma_{\varepsilon}^{2}}
$$

where

$$
\frac{\partial \operatorname{var}(\pi \mid e=1)}{\partial \sigma_{\varepsilon}^{2}}=\Gamma_{1}\left[\Gamma_{1}-\frac{2 \alpha \sigma_{\eta}^{2}\left(\sigma_{\eta}^{2}+\sigma_{\varepsilon}^{2}\right)}{\left(\sigma_{\varepsilon}^{2}+\sigma_{\eta}^{2}\right)^{2}}\right]=\Gamma_{1}\left[\beta^{*}-\frac{\alpha \sigma_{\eta}^{2} \sigma_{\varepsilon}^{2}}{\left(\sigma_{\varepsilon}^{2}+\sigma_{\eta}^{2}\right)^{2}}\right]
$$

Q.E.D.

While this condition is not particularly intuitive, examining this more closely yields the next proposition which constitutes the main contention of the paper.

Proposition 2 The agency cost $w_{1}$ decreases with the extent of uncertainty $\sigma_{\varepsilon}^{2}$ for any given $\left(c, \alpha, \sigma_{\eta}^{2}, \sigma_{\varepsilon}^{2}\right)$ if $\mu$ is sufficiently large.

PROOF: We first show that $\partial \operatorname{var}(\pi \mid e=1) / \partial \sigma_{\varepsilon}^{2}<0$ is actually a sufficient condition for $\partial w_{1} / \partial \sigma_{\varepsilon}^{2}<$ 0. To see this, note that $\beta^{*}$ must satisfy

$$
\beta^{*}=\frac{c}{\mu}+\frac{r}{2 \mu}[\operatorname{var}(\pi \mid e=1)-\operatorname{var}(\pi \mid e=0)]=\frac{c}{\mu}+\frac{r}{2 \mu}\left[\operatorname{var}(\pi \mid e=1)-\beta^{* 2} \sigma_{\varepsilon}^{2}\right] .
$$

It follows from this that an increase in $\sigma_{\varepsilon}^{2}$ decreases the righthand side with $\beta^{*}$ held constant if $\partial \operatorname{var}(\pi \mid e=1) / \partial \sigma_{\varepsilon}^{2}<0$. Since $\beta^{*}$ is the smaller root of (17) (see the proof of lemma 2 for more on this), this also implies $\partial \beta^{*} / \partial \sigma_{\varepsilon}^{2}<0$, proving that $\partial \operatorname{var}(\pi \mid e=1) / \partial \sigma_{\varepsilon}^{2}$ is actually a sufficient condition for $\partial w_{1} / \partial \sigma_{\varepsilon}^{2}<0$. More precisely, given some optimal contract $\left(\beta^{*}\left(\sigma_{\eta}^{2}, \sigma_{\varepsilon}^{2}\right), \gamma^{*}\left(\sigma_{\eta}^{2}, \sigma_{\varepsilon}^{2}\right)\right)$, the agency cost decreases with $\sigma_{\varepsilon}^{2}$, evaluated at $\sigma_{\varepsilon}^{2}$, if

$$
\beta^{*}\left(\sigma_{\eta}^{2}, \sigma_{\varepsilon}^{2}\right)<\frac{\alpha \sigma_{\eta}^{2} \sigma_{\varepsilon}^{2}}{\left(\sigma_{\varepsilon}^{2}+\sigma_{\eta}^{2}\right)^{2}}
$$

This condition holds for any given $\left(c, \alpha, \sigma_{\eta}^{2}, \sigma_{\varepsilon}^{2}\right)$ when $\mu$ is sufficiently large since $\lim _{\mu \rightarrow \infty} \beta^{*}=0$.

Q.E.D.

A straightforward way to interpret this result is that the standard tradeoff between risk and incentives tends to disappear when the agent is more self-confident. This interpretation should be 
made with caution, however, because what a high $\mu$ means is only relative, especially to the cost of performing the task $c$. Our preferred view is slightly different, though closely related. Fixing $c$ constant, an increase in $\mu$ alternatively implies that the task at hand is getting easier or less costly, and weaker incentives are needed to induce effort (the optimal contract converging to a fixed-wage scheme as $\mu \rightarrow \infty)$. This means that the agent can earn less pecuniary rents by performing the task, consequently rendering the self-esteem concern more significant in a relative sense. This implication thus leads to a potentially testable hypothesis: the tradeoff between risk and incentives becomes more ambiguous when expected material rents are relatively small. Conversely, it is also expected that the standard tradeoff becomes more visible for those with more lucrative contracts, such as CEOs and professional athletes who can supposedly earn larger rents. Indeed, according to the evidence presented by Prendergast (2002), the relationship between risk and incentives is ambiguous at best for CEOs while there is a clear positive relationship for sharecroppers and franchisees.

Although more empirical studies along this line are certainly needed before we jump to a firm conclusion, this argument seems to reveal something fundamental about any explanations that appeal to psychological factors. Since psychological factors are normally exogenously given and the magnitude of their effects is roughly fixed, those factors tend to be more accentuated when less material benefits are at stake. ${ }^{13}$ For instance, it is well known in social psychology that incentives (explicit rewards) may backfire as they often interfere with 'intrinsic motivation.' ${ }^{14}$ Subsequent studies reveal that this anomaly, which certainly contradicts with the conventional economic reasoning, is more likely to arise when material benefits are not sufficient (e.g., Gnnezy and Rustichini, 2000). In other words, any effect that stems from exogenously fixed psychological factors is more resounding when less is at stake materially but tends to be blown away in the face of significant material incentives. A similar logic certainly works in the present model: when less is at stake materially, the agent's attention naturally shifts towards psychological factors such as self-esteem concerns, which substantially weaken or even

\footnotetext{
${ }^{13}$ This is especially true when the consumption value of self-esteem is more dominant because it is reasonable to regard its effect as exogenously fixed.

${ }^{14}$ One of the famous examples is an observation by Titmus (1970) that paying for blood would reduce the willingness to donate blood as it undermines its social value.
} 
overturn the prediction made by the standard economic theory.

Besides this implication, the present model also sheds light on two key ingredients of the main result, each of which yields a potentially testable hypothesis. First, we assume at the outset that ability and effort are complementary to each other, which is crucial in giving rise to the need for selfhandicapping. In fact, the conclusion is totally reversed when they are substitutive, i.e., when only one of either ability or effort is sufficient to carry out a task. In that case, the agent actually exposes himself less to relevant information by exerting effort since more effort can cover the lack of ability. Note that this need to hide the lack of ability diminishes as the underlying environment becomes noisier and the outcome loses its informational content. The model thus predicts an important, possibly testable, connection between the complementarity between ability and effort on the one hand and the tradeoff between risk and incentives on the other: the standard tradeoff becomes less visible in situations where effort and ability are more complementary.

Second, the way we model self-esteem concerns implicitly assumes that the agent is risk-averse in his own self-images. In other words, we implicitly assume by design that the agent is relatively averse to receiving a bad news about himself (which may be referred to as 'information-aversion'). This condition is critical because the conclusion is once again totally overturned if the agent is riskloving in his own self-images. Suppose that this is the case so that the agent is actually made better off by collecting more accurate information about himself, given the same mean. In this case, besides monetary compensations, the agent has an additional incentive to 'discover himself' when he faces a task. This extra incentive is naturally stronger when the underlying situation involves less uncertainty as the agent is likely to obtain a more accurate signal in that case. The effect arising from self-esteem concerns then works in the same direction as the standard tradeoff: an increase in uncertainty diminishes this extra incentive, thereby increasing the agency cost. This reasoning implies that information-aversion (or truth-aversion) constitutes a necessary component for our story to be valid when it is combined with the complementarity between ability and effort. ${ }^{15}$

\footnotetext{
${ }^{15}$ The standard tradeoff also breaks down when the agent is risk-loving in his own self-images, and ability and effort are substitutive. We do not explore much on this case since this combination seems to be less likely.
} 
Although this assumption of information-aversion seems to be a reasonable specification for many cases, as exemplified by several episodes of self-handicapping behavior, this implication indicates that the question of how we value self-esteem (the sign of the second derivative), instead of just whether we care about it (the sign of the first derivative), can be a critical agenda on this issue.

\subsection{A numerical example}

We conclude the section with a numerical example in order to present a clearer picture of the model. More specifically, we are interested in how the optimal incentive $\beta^{*}$ and the agency cost $w_{1}$ vary with the extent of uncertainty $\sigma_{\varepsilon}^{2}$ for given values of $\sigma_{\eta}^{2}$ and $\alpha .^{16}$

Figures 1a and 1b illustrate $\beta^{*}$ for $\alpha=0.75$ and $\alpha=1$. In each figure, three different values of $\sigma_{\eta}^{2}$ are considered. As the figures indicate, the optimal incentive $\beta^{*}$ decreases with $\sigma_{\varepsilon}^{2}$ for a wide range of $\sigma_{\varepsilon}^{2}$. Indeed, the optimal incentive decreases rather sharply when $\sigma_{\varepsilon}^{2}$ is relatively small. This tendency is clearly more visible when the self-esteem concerns is stronger $(\alpha=1)$.

[figures $1 \mathrm{a}$ and $1 \mathrm{~b}$ about here]

Figures $2 \mathrm{a}$ and $2 \mathrm{~b}$ illustrate how the agency cost $w_{1}$ varies with $\sigma_{\varepsilon}^{2}$. The figures confirm that there indeed exists some range for which the agency cost does decrease with $\sigma_{\varepsilon}^{2}$, due mostly to a decrease in $\beta^{*}$. They also indicate that the agency cost becomes larger when the self-esteem concern is strong (a large $\alpha$ ).

[figures $2 \mathrm{a}$ and $2 \mathrm{~b}$ about here]

The figures above also indicate that the stronger incentive is necessary and the agency cost is larger for more fragile individuals (one with a larger $\sigma_{\eta}^{2}$ ), illustrating an inherent relationship that exists between the self-esteem fragility $\sigma_{\eta}^{2}$ and its level $\mu$. The reason for why fragile self-esteem is not desirable for the principal is intuitively clear and simple: more fragile individuals face more uncertainly both for the outcome and the posterior belief and hence demand more to compensate for this loss.

\footnotetext{
${ }^{16}$ For the example, we specify $r=1, c=0.1$ and $\mu=2$.
} 
This indicates that when it comes down to selecting an agent (say, a worker), how fragile (or secure) the individual is about his own self-images is a factor just as crucial as his expected productivity, e.g., the expected ability.

\section{$5 \quad$ Why and how can team production be profitable?}

It has recently been suggested that many firms adopt team production as a means to motivate workers and improve productivity. This is somewhat puzzling, at least on purely theoretical grounds, because team production invites the free-rider problem in standard moral hazard problems. In order to account for this gap, several explanations have been offered. An obvious explanation is to point to the presence of complementarities or synergies among team members. A benefit of team production also arises in a long-term relationship where team members interact with each other repeatedly over time. ${ }^{17}$

We intend to offer an alternative view on a virtue of team production, with an emphasis on the role of self-esteem concerns. Since a failure can be attributed at least partially to the teammates, team production inevitably introduces ambiguity into the self-evaluation process. It is therefore less ego-threatening to exert effort under team production than under individual production because the outcome now reflects the teammates' attributes as well as the agent's own, and hence they can share the blame in the case of an unsuccessful event.

For the sake of comparison, we consider exactly the same specification as in the previous section. The only difference is that the principle now hires two agents, denoted by $i=A, B$, and lets them perform a task jointly. The only available measure of performance is the aggregate outcome $y$, which is given by

$$
y=\frac{\left(\eta_{A} e_{A}+\varepsilon_{A}\right)+\left(\eta_{B} e_{B}+\varepsilon_{B}\right)}{2}
$$

where $\varepsilon_{i}$ is i.i.d. This additive nature of the production function implies that there are no technological complementarities between the agents, a potential source of the benefit of team production.

Under this setup, we show that the principle may induce effort $e_{i}=1$ from both agents at a lower

\footnotetext{
${ }^{17}$ See Che and Yoo (2001) and Ishida (2006) for this approach.
} 
cost when they work jointly. To see this, it is once again crucial to obtain the variance of the total payoff $\pi_{i}$. The following result is obtained by slightly modifying lemma 1.

\section{Lemma 4}

$$
\operatorname{var}\left(\pi_{i} \mid e_{i}, e_{j}\right)=\left(\frac{\beta e_{i}}{2}+\frac{\alpha e_{i}^{2} \sigma_{\eta}^{2}}{2 \sigma_{\varepsilon}^{2}+e_{i}^{2} \sigma_{\eta}^{2}}\right)^{2} \sigma_{\eta}^{2}+2\left(\frac{\beta}{2}+\frac{\alpha e_{i} \sigma_{\eta}^{2}}{2 \sigma_{\varepsilon}^{2}+e_{i}^{2} \sigma_{\eta}^{2}}\right)^{2} \sigma_{\varepsilon}^{2}+\left(\frac{\beta e_{j}}{2}\right)^{2} \sigma_{\eta}^{2}, i \neq j
$$

PROOF: The result obtained here largely parallels to lemma 1 . The outcome under team production contains some information about the teammate's ability type, which functions as a noise in the selfevaluation process. The best estimate from the aggregate outcome is $\left(2 y-\mu e_{j}\right) / e_{i}=\left(\eta_{i} e_{i}+\varepsilon_{i}+\right.$ $\left.\varepsilon_{j}\right) / e_{i}:=\hat{\eta}_{i}, i \neq j$. Note that $\hat{\eta}_{i}$ is distributed normally with precision $e^{2} / 2 \sigma_{\varepsilon}^{2}$. The posterior belief is then given by

$$
\tilde{\mu}_{i}=\frac{\frac{1}{\sigma_{\eta}^{2}} \mu+\frac{e^{2}}{2 \sigma_{\varepsilon}^{2}} \hat{\eta}_{i}}{\frac{1}{\sigma_{\eta}^{2}}+\frac{e_{i}^{2}}{2 \sigma_{\varepsilon}^{2}}}=\frac{2 \sigma_{\varepsilon}^{2} \mu+e_{i}^{2} \sigma_{\eta}^{2} \hat{\eta}_{i}}{2 \sigma_{\varepsilon}^{2}+e_{i}^{2} \sigma_{\eta}^{2}}=\frac{2 \sigma_{\varepsilon}^{2} \mu+e_{i}^{2} \sigma_{\eta}^{2} \eta_{i}+e_{i} \sigma_{\eta}^{2}\left(\varepsilon_{i}+\varepsilon_{j}\right)}{2 \sigma_{\varepsilon}^{2}+e_{i}^{2} \sigma_{\eta}^{2}} .
$$

It then follows from this that the agent's ex post payoff is

$$
\pi_{i}=\beta \frac{\left(\eta_{i} e_{i}+\varepsilon_{i}\right)+\left(\eta_{j} e_{j}+\varepsilon_{j}\right)}{2}+\gamma-c\left(e_{i}\right)+\alpha \frac{e_{i}^{2} \sigma_{\eta}^{2}\left(\eta_{i}-\mu\right)+e_{i} \sigma_{\eta}^{2}\left(\varepsilon_{i}+\varepsilon_{j}\right)}{2 \sigma_{\varepsilon}^{2}+e_{i}^{2} \sigma_{\eta}^{2}} .
$$

Since $\alpha, \gamma$ and $\mu$ are all constant at this stage, we have

$$
\operatorname{var}\left(\pi_{i} \mid e_{i}, e_{j}\right)=\left(\frac{\beta e_{i}}{2}+\frac{\alpha e_{i}^{2} \sigma_{\eta}^{2}}{2 \sigma_{\varepsilon}^{2}+e_{i}^{2} \sigma_{\eta}^{2}}\right)^{2} \sigma_{\eta}^{2}+2\left(\frac{\beta}{2}+\frac{\alpha e_{i} \sigma_{\eta}^{2}}{2 \sigma_{\varepsilon}^{2}+e_{i}^{2} \sigma_{\eta}^{2}}\right)^{2} \sigma_{\varepsilon}^{2}+\left(\frac{\beta e_{j}}{2}\right)^{2} \sigma_{\eta}^{2} .
$$

Q.E.D.

Given this, the incentive compatibility constraint (for agent $A$ ) is

$$
\frac{\beta \mu}{2} \geq c+\frac{r}{2}\left[\operatorname{var}\left(\pi_{A} \mid e_{A}=1, e_{B}\right)-\operatorname{var}\left(\pi_{A} \mid e_{A}=0, e_{B}\right)\right],
$$

while the participation constraint is

$$
\beta \mu \frac{e_{A}+e_{B}}{2}+\gamma \geq c\left(e_{A}\right)+\frac{r}{2} \operatorname{var}\left(\pi_{A} \mid e_{A}, e_{B}\right)
$$

Define $\beta^{* *}$ as the minimum incentive needed to induce effort $e_{A}=e_{B}=1$ from both agents which must satisfy

$$
\beta^{* *}=\frac{2 c}{\mu}+\frac{r}{\mu}\left[\left(\frac{\beta}{2}+\frac{\alpha \sigma_{\eta}^{2}}{2 \sigma_{\varepsilon}^{2}+\sigma_{\eta}^{2}}\right)^{2}\left(2 \sigma_{\varepsilon}^{2}+\sigma_{\eta}^{2}\right)-2\left(\frac{\beta}{2}\right)^{2} \sigma_{\varepsilon}^{2}\right] .
$$


The agency cost of inducing $e_{A}=e_{B}=1$ under team production is then defined as

$$
W_{1}\left(\sigma_{\eta}^{2}, \sigma_{\varepsilon}^{2}\right)=c+\frac{r}{2}\left[\left(\frac{\beta^{* *}\left(\sigma_{\eta}^{2}, \sigma_{\varepsilon}^{2}\right)}{2}+\frac{\alpha \sigma_{\eta}^{2}}{2 \sigma_{\varepsilon}^{2}+\sigma_{\eta}^{2}}\right)^{2}\left(2 \sigma_{\varepsilon}^{2}+\sigma_{\eta}^{2}\right)+\left(\frac{\beta^{* *}\left(\sigma_{\eta}^{2}, \sigma_{\varepsilon}^{2}\right)}{2}\right)^{2} \sigma_{\eta}^{2}\right] .
$$

Comparing $W_{1}$ with $w_{1}$ obtained in the last section, we can make the following claim which is very similar in nature to proposition 2.

Proposition 3 The agency cost is lower under team production than under individual production, i.e., $W_{1}\left(\sigma_{\eta}^{2}, \sigma_{\varepsilon}^{2}\right)<w_{1}\left(\sigma_{\eta}^{2}, \sigma_{\varepsilon}^{2}\right)$, for any given $\left(c, \alpha, \sigma_{\eta}^{2}, \sigma_{\varepsilon}^{2}\right)$ if $\mu$ is sufficiently large.

Proof: The agency cost decreases under team production if $\operatorname{var}\left(\pi_{i} \mid e_{A}=1, e_{B}=1\right)<\operatorname{var}(\pi \mid e=$ 1), i.e.,

$$
\left(\frac{\beta^{* *}\left(\sigma_{\eta}^{2}, \sigma_{\varepsilon}^{2}\right)}{2}+\frac{\alpha \sigma_{\eta}^{2}}{2 \sigma_{\varepsilon}^{2}+\sigma_{\eta}^{2}}\right)^{2}\left(2 \sigma_{\varepsilon}^{2}+\sigma_{\eta}^{2}\right)+\left(\frac{\beta^{* *}\left(\sigma_{\eta}^{2}, \sigma_{\varepsilon}^{2}\right)}{2}\right)^{2} \sigma_{\eta}^{2}<\left(\beta^{*}+\frac{\alpha \sigma_{\eta}^{2}}{\sigma_{\varepsilon}^{2}+\sigma_{\eta}^{2}}\right)^{2}\left(\sigma_{\varepsilon}^{2}+\sigma_{\eta}^{2}\right) .
$$

Note that $\lim _{\mu \rightarrow \infty} \beta^{*}=0$ and $\lim _{\mu \rightarrow \infty} \beta^{* *}=0$. As $\mu \rightarrow \infty$, therefore, (27) can be written as

$$
\left(\frac{\alpha \sigma_{\eta}^{2}}{2 \sigma_{\varepsilon}^{2}+\sigma_{\eta}^{2}}\right)^{2}\left(2 \sigma_{\varepsilon}^{2}+\sigma_{\eta}^{2}\right)<\left(\frac{\alpha \sigma_{\eta}^{2}}{\sigma_{\varepsilon}^{2}+\sigma_{\eta}^{2}}\right)^{2}\left(\sigma_{\varepsilon}^{2}+\sigma_{\eta}^{2}\right)
$$

which can be shown to hold for any given $\left(c, \alpha, \sigma_{\eta}^{2}, \sigma_{\varepsilon}^{2}\right)$.

Q.E.D.

There are now increasingly many firms that adopt internal team competition in various formats as a means to motivate workers. Many managers and executives emphasize the psychological side of team production although it is not entirely clear through what channel team production can provide so much motivation to team members, especially to the extent that can overcome the free-rider problem. The present model suggests one possible channel through which that can happen: workers are better motivated under team production because ambiguity inherent under team production is instrumental in mitigating the need for self-handicapping. Although we do not necessarily claim that this factor alone is strong enough to make team production more effective than individual production, it can be a factor that plays at least some role in it. 


\section{Concluding remarks}

This paper examines the role of self-esteem concerns in contracting situations via a simple model of moral hazard. When the agent is relatively averse to having a negative self-image, there arises an incentive to remain ignorant about himself, ultimately amounting to the need for self-handicapping. In this situation, uncertainty may have a positive incentive effect as it introduces ambiguity into the self-evaluation process. This actually mitigates the need for self-handicapping.

The main purpose of the paper is simply to illustrate the point that the presence of self-esteem concerns yields an implication that goes against the conventional wisdom. For this reason, there are several avenues to extend the present analysis. First, it is an important extension to examine the role of self-esteem concerns in more general settings, e.g., continuous effort choices and/or more general, nonlinear, contracts. Of course, as is well known, it is hard enough to generally characterize the optimal contract in moral hazard problems even without self-esteem concerns. It is nonetheless an

important agenda to see how far one can push this logic and generalize the results obtained in the present paper.

Second, this paper assumed that the agent in question is a rational Bayesian learner who can objectively compute his posterior belief. In reality, though, it is well known that people often resort to ways to manipulate their own beliefs, from self-serving bias and cognitive dissonance to downward comparison. For instance, self-serving bias is another inherent human tendency which is though to be a part of the self-defence mechanism. Although we emphasize the aspect that the main results hold even for rational Bayesian learners, it is of some interest to see interactions, if any, between self-esteem concerns and biased information processing.

\section{References}

Ackerberg, Daniel A. and Maristella Botticini. 2002. Endogenous Matching and the Empirical Determinants of Contract Form. Journal of Political Economy 110 (June): 564-91.

Bandura, Albert. 1977. Self-Efficacy: Toward a Unifying Theory of Behavioral Change. Psychological 
Review 84 (March): 191-215.

Barkow, Jerome H. 1980. Prestige and Self-Esteem: A Biosocial Interpretation. In Omerk, Donald R, Frederick F. Strayer and Daniel G. Freedman (eds.), Dominance Relations, New York: Garland.

Benabou, Roland and Jean Tirole. 2002. Self-Confidence and Personal Motivation. Quarterly Journal of Economics 117 (August): 871-915.

. 2003. Intrinsic and Extrinsic Motivation. Review of Economic Studies 62 (April): 315-39. . 2004. Willpower and Personal Rules. Journal of Political Economy 112 (August): 847-887. . 2006. Incentives and Prosocial Behavior. forthcoming in American Economic Review.

Berglas, Steven and Edward E. Jones. 1978. Drug Choice as Self-Handicapping Strategy in Response to Success. Journal of Personality and Social Psychology 36: 405-17

Carrillo, Juan D. and Thomas Mariotti. 2000. Strategic Ignorance as a Self-Disciplining Device. Review of Economic Studies 67 (July): 529-44.

Che, Yeon-Koo and Yoo, Seung-Weon. 2001. Optimal Incentives for Teams. American Economic Review 91 (June): 525-41.

Core, John E. and Wayne R. Guay. 2002. The Other Side of the Trade-Off: the Impact of Risk on Executive Compensation: A Revised Comment. mimeo. University of Pennsylvania.

DeGroot, Morris H. 1970. Optimal Statistical Decisions, New York: McGraw-Hill.

Dewatripont, Mathias; Ian Jewitt and Jean Tirole. 1999a. The Economics of Career Concerns, Part I: Comparing Information Structures. Review of Economic Studies 66 (January): 183-98. . 1999b. The Economics of Career Concerns, Part II: Applications to Missions and Accountability of Government Agencies. Review of Economic Studies 66 (January): 199-217.

Englmaier, Florian and Achim Wambach. 2006. Optimal Incentive Contracts under Inequality Aversion. mimeo. 
Gneezy, Uri and Aldo Rustichini. 2000. Pay Enough or Don't Pay at All. Quarterly Journal of Economics 115 (August): 791-810.

Greenberg, Jeff; Tom Pyszczynski and Sheldon Solomon. 1986. The Causes and Consequences of the Need for Self-Esteem: A Terror Management Theory. In Baumeister, Roy F. (ed.) Public Self and Private Self, New York: Springer-Verlag.

Grund, Christian and Dirk Sliwka. 2005. Envy and Compassion in Tournaments. Journal of Economics and Management Strategy 14 (Spring): 187-207.

Holmstrom, Bengt. 1999. Managerial Incentive Problems: A Dynamic Perspective. Review of Economic Studies 66 (January): 169-82.

Ishida, Junichiro. 2006. Team Incentives under Relative Performance Evaluation. Journal of Economics and Management Strategy 15 (March): 187-206.

. 2006. Optimal Promotion Policies with the Looking-Glass Effect. Journal of Labor Economics 24 (October): 857-77.

Itoh, Hideshi. 2004. Moral Hazard and Other-Regarding Preferences. Japanese Economic Review 55 (March): 18-45.

Jin, Li. 2002. CEO Compensation, Diversification and Incentives. Journal of Financial Economics 2002 (October): 29-63.

Koszegi, Botond. 2006. Ego Utility, Overconfidence and Task Choice. forthcoming in Journal of the European Economic Association.

Leary, Mark R. and Downs, Deborah L. 1995. Interpersonal Functions of the Self-Esteem Motive: the Self-Esteem System as a Sociometer. In Kernis, Michael H. (ed.), Efficacy, Agency, and SelfEsteem, New York: Plenum.

McFarlin, Dean B.; Roy F. Baumeister and Jim Blascovich. 1984. On Knowing When to Quit: Task Failure, Self-Esteem, Advice and Non-Productive Persistence. Journal of Personality 52 (June): 
138-55.

Prendergast, Canice. 2000. What Trade-Off of Risk and Incentives? American Economic Review 90 (May): 421-25.

. 2002a. The Tenuous Tradeoff between Risk and Incentives. Journal of Political Economy 110 (October): 1071-102. . 2002b. Uncertainty and Incentives. Journal of Labor Economics 20 (April): S115-34.

Raith, Michael. 2003. Competition, Risk and Manegerial Incentives. American Economic Review 93 (September): 1425-36.

Tesser, Abraham. 2001. Self-Esteem. In Tesser, Albert and Norbert Schwarz (eds.), Handbook of Social Psychology: Intraindividual Processes, Malden, MA: Blackwell.

Tesser, Abraham and James E. Collins. 1988. Emotion in Social Reflection and Comparison Situations: Intuitive, Systematic and Exploratory Approaches. Journal of Personality and Social Psychology 55 (November): 695-709.

Tesser, Abraham and Leonard L. Martin. 1996. The Psychology of Evaluation. In Higgins, E. Tory and Arie W. Kruglanski (eds.), Social Psychology: Handbook of Basic Principles, New York: Guilford Press.

Titmus, Richard M. 1970. The Gift Relationship, London: Allen and Unwin.

Tucker, Jalie A.; Rudy E. Vuchinich and Mark B. Sobel. 1981. Alcohol Consumption as a SelfHandicapping Strategy. Journal of Abnormal Psychology 90 (June): 220-30. 


\section{Figures}

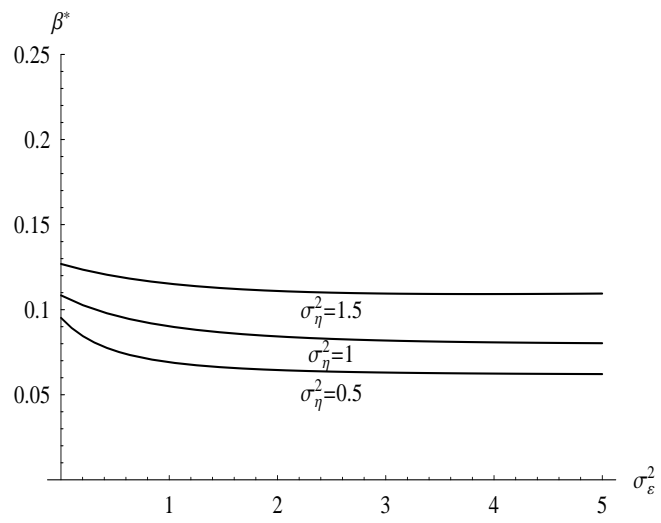

Figure 1a: $\alpha=0.75$

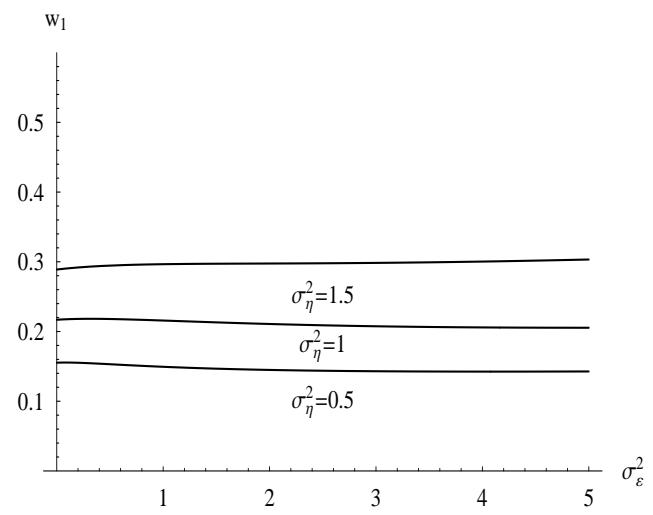

Figure 2a: $\alpha=0.75$

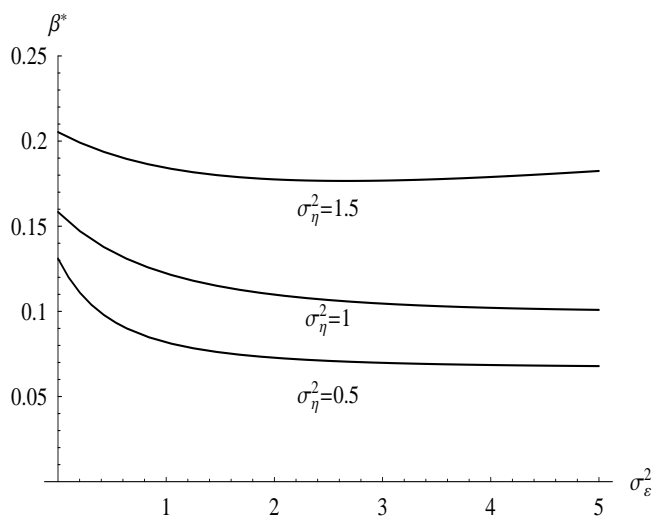

Figure 1b: $\alpha=1$

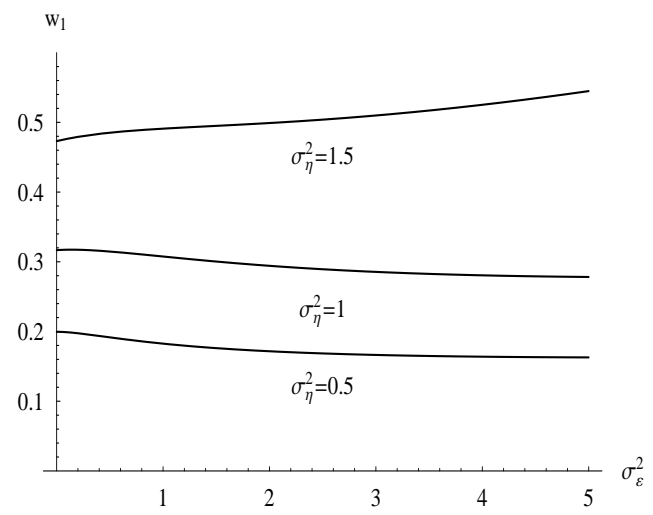

Figure 2b: $\alpha=1$ 